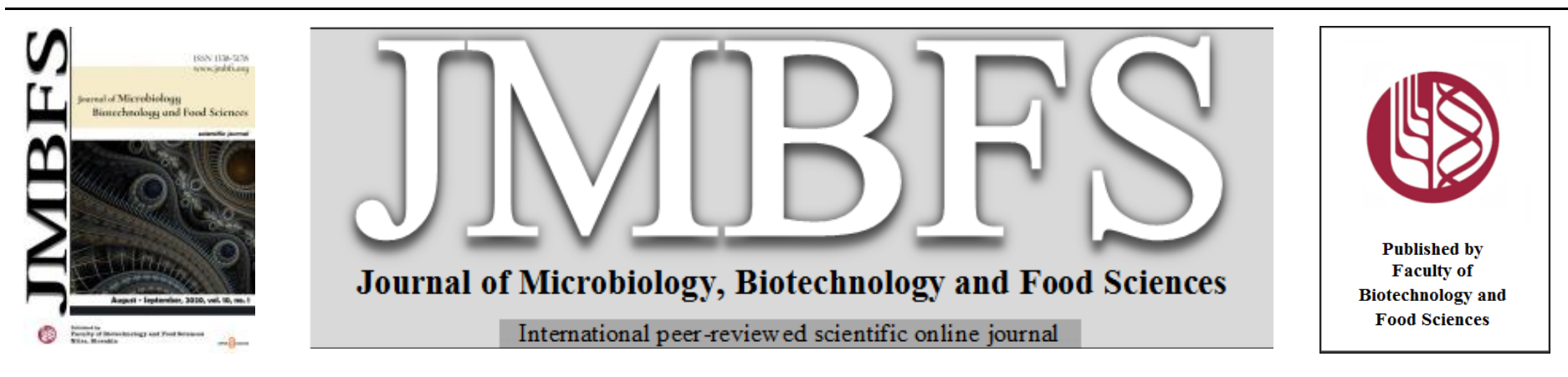

\title{
NATIVE PLANT GROWTH PROMOTING RHIZOBACTERIA WITH REMARKABLE PHORATE METABOLISING ABILITIES AT CONCENTRATIONS MULTI-FOLD HIGHER THAN RESIDUAL CONCENTRATION PRESENT IN SOIL
}

\section{Pratibha Yadav and S Krishna Sundari*}

Address(es): Prof. S. Krishna Sundari,

Jaypee Institute of Information Technology (JIIT), A-10, Sector: 62, 201309, NOIDA, U.P., India, 91-120-2594206.

*Corresponding author: krishna.sundari@jiit.ac.in, skrishnasundari@ gmail.com

doi: 10.15414/jmbfs.2020.10.1.54-60

\section{ARTICLE INFO}

Received 11.2. 2019

Revised 11.3. 2020

Accepted 11.3. 2020

Published 1. 8. 2020

Regular article open $\mathcal{O}$ access

\begin{abstract}
Native rhizobacteria were isolated from agriculture soil and evaluated for their pesticide remediation potential. Native rhizobacterial isolates (RBI1, RBI2, RBI3 and RBI4) employed in the study exhibited high levels of tolerance towards phorate (10\% CG) registering MIC (minimum inhibitory concentration) values, between 1450-2010 ppm. The isolates RBI1, RBI4 exhibited effeciency (35\%-87\%) to catabolise/co-metabolise phorate as sole energy (ClP) source at concentration as high as $500 \mathrm{ppm}$. FTIR analysis suggested evidence for degradtion of complex parent compound (phorate) to less complex degradtion metabolites. Disappearance of specific ester linkages in control $\left(1025.43,998.37\right.$ and $\left.908.99 \mathrm{~cm}^{-1}\right)$ suggested hydrolysis of ester bonds, which is a sure indication of organophosphate's degrdation. Phorate degradation by native isolate was further quantified through HPLC analysis presenting $42 \%$ degradation (within 48hrs) at concentrations 10-15 fold higher (300 ppm) as compared to residual phorate reported in soils. Current study is the very first report providing a biologically safe option of rhizoremediation to degrade higher concentrations of persistent phorate residues, at concentrations rarely explored thus far. Thus the study provides substantial evidence regarding the potential of rhizobacterial isolates to be developed as bioinoculants and applied for accelerated remediation of toxic phorate residues.
\end{abstract}

\section{INTRODUCTION}

The IUPAC nomenclature of phorate is O-diethyl-S-(ethylthio) methylphosphorodithioate. It is a second generation pesticide belonging to the organophosphate pesticide (OPP) group and is a broad spectrum systemic insecticide. Phorate is available both in emulsion and granular forms and considered as most active insecticide applied to control pests such as beetles, borers, leaf worms, maggots, nematodes, termites, mites, rootworms, etc Phorate usage is registered with the central insecticide board and registration committee (CIBRC), India, to control pests in several crops such as sorghum, corn, sugarcane, bajara, soybean, maize, tomato, beans, potatoes, peanuts etc (Bano and Musarrat, 2003; Singh et al., 2003; Maria, 2010; Gangadhar et al., 2016). In the past 40 years organophosphate pesticides have found increasing application. Total phorate production was $4800 \mathrm{MT}$ and average field application ranging between 7.5 to $10 \mathrm{~kg}$ per acre in India ( Rani $\boldsymbol{e t}$ al., 2009; Gupta et al., 2015; Jariyal et al., 2015). Depending on the environmental conditions phorate has a half life of 2 - 173 days in soil and remains persistent for longer time in different soil systems. As phorate interacts with soil particles and gets adsorbed easily to soil surface, makes itself unavailable for natural degradation processes thus leading to its persistence is enhanced. Rate of phorate breakdown/degradation mostly depends upon soil microbial diversity, soil nutrient composition and soil microclimate. Phorate is water soluble $(50 \mathrm{mg} / \mathrm{lit})$ and as a result it readily leaches out contaminating water bodies including ground water. From these polluted water bodies its trace amount re-enters farmlands through different irrigation mechanism. Phorate is reported as one of the most toxic pesticide, with average $\mathrm{LD}_{50}$ value of $2-4 \mathrm{mg} / \mathrm{kg}$ (Jariyal et al., 2018).

Studies reporting presence of target pesticide residues in agricultural products is a pressing concern and solutions for safe breakdown of these pesticides are the need of the hour (Gevao et al., 2000; Yadav et al., 2015; Chaudhary and Shukla, 2019). Many studies have identified high phorate toxicity in non-target organisms harming land and aquatic life (Morowati, 1998; Berny et al., 2010; Abdollahi and Karami-Mohajeri, 2012). Predicted lethal dosage of phorate for humans (upon ingestion) was observed to be less than $5 \mathrm{ppm} / \mathrm{kg}$ body weight. World Health Organisation (WHO) has classified phorate as "highly toxic" chemical as it irreversibly inhibits an important enzyme in human body namely acetylcholinesterase (ACE) at concentrations as low as 1 ppm (US EPA, 1998) Inhibition of ACE impairs transmission of nerve impulses causing serious neurological deformities (Antonijevic and Stojiljkovic, 2007; Rani et al., 2009; Karami-Mohajeri and Abdollahi, 2011; Jariyal et al., 2015).

Bioremediation techniques are practiced vastly to detoxify persistent organic pollutants including pesticides. Microbe assisted degradation of complex organophosphate pesticides is a currently evolving research area (Sørensen $\boldsymbol{e t}$ al., 2008; RatnaKumari et al., 2012; El-Helow et al., 2013; Chaudhary and Shukla, 2019). Bacteria belonging to species: Pseudomonas, Bacillus, Brevibacterium, Ralstonia, Staphylococcus and Flavobacterium that produce important degradation enzymes such as phosphatase, esterase and other hydrolytic enzymes were found to be involved in pesticide breakdown (Venkatramesh et al., 1987; Kadam and Gangawane, 2005; Singh and Walker, 2006; Rani et al., 2009; Rani and Juwarkar, 2010; Rani and Juwarkar, 2012, Jariyal et al., 2015). Fewer research studies addressed phorate bioremediation as compared to other OPP's such as: chloripyrifos, methyl parathion, parathion etc. Many authors reported greater toxicity of phorate degradation metabolites like phosphodithioate sulfoxide and phosphodithioate sulfone that may impact the degradative potential of active micro flora Moreover, some species reported so far for phorate degradation were suspected human pathogens, (viz., Pseudomonas aeuroginosa and Enterobacter cloacae) thus limiting their development as field applicable formulations. Bio formulations with opportunistic pathogen may cause occupational exposure in farmers, resulting in operational risks and hazard. Such microbes cannot be applied for remediation of pesticide residues in open fields on a large scale. Additionally, supplementing the soil with such possible pathogenic microbes targeting a specific pollutant may tilt the microbial diversity in agriculture soil challenging balanced plant growth. In addition, any suggestion of real time microbe assisted pesticide degradation should address the relationship of such microbes with plants and their ability to support plant growth.

Existing literature for microbe assisted degradation of phorate does not offer a clear choice of candidate microbes that are safe to use, tolerate high phorate concentrations and also support plant growth. These observations have lead our research in the present direction where we have begun our study employing isolates from author's collection that were previously tested for their ability to 
produced biosurfactants, bio-emulsifiers and biofilm to assist in accelerated degradation of organophosphate pesticides. Authors reported the plant growth supporting ability of these isolates and found that they were able to improve growth of plant host Vigna radiata in terms of \% germination, seed vigour and total biomass (Yadav and Sundari, 2015). In this study we have evaluated the degradative potential of four rhizobacterial isolates to metabolise phorate at concentrations starting from $100 \mathrm{ppm}$ till $500 \mathrm{ppm}$ and their tolerance to concentrations greater than $1000 \mathrm{ppm}$. Substrate exclusion studies were conducted to evaluate whether the test isolates can metabolise phorate either as sole carbon or sole phosphate source. Degradation of phorate by test isolates was further validated by FTIR and HPLC analysis. Establishing the ability of native rhizobacterial isolates through the present manuscript can be an important value addition to design microbe assisted degradation strategies for on-site remediation of pesticide residues in future.

\section{MATERIAL AND METHODS}

\section{Preparation of Pesticide stock}

Market available Phorate formulation (Phoratox-10, Phorate 10\% CG encapsulated) was used in the current microbe assisted degradation studies. A stock solution of $10 \mathrm{mg} / \mathrm{ml}$ of phorate was prepared and maintained at $4^{\circ} \mathrm{C}$ Specific concentrations of phorate were freshly prepared from time to time using stock solution as per the requirement of individual experiments.

\section{Preparation of bacterial inoculum}

A total of four rhizobacteria were, isolated from rhizhospheric soils of agricultural fields (near Narora, Dist. Bulandshahar, U.P., India). The isolates were purified after enrichment culture on CRA and King's B broth supplemented with $100 \mathrm{ppm}$ of phorate (Rani et al., 2009; Jariyal et al., 2015; Yadav and Sundari, 2015). These isolates served as the test isolates in the present study. Isolates RBI1 and RBI2 were maintained on nutrient agar where as RBI3 and RBI4 on King's B agar. The isolates were characterized based on biochemical phenotypic and molecular traits. Nomenclature of the isolates was reported by authors (paper communicated). For all the degradation experiments conducted, an individually inoculated overnight grown culture of respective isolates containing $2 \times 10^{-7} \mathrm{cfu} / \mathrm{ml}$ was used as inoculum. All experimental treatments were placed in triplicates and experiments repeated twice for checking repeatability of results. Data was analysed using Windows 8 Excel software.

\section{Phorate degradation studies}

\section{Tolerance to Pesticide}

Rhizobacterial isolates RBI1, RBI2, RBI3 and RBI4 were tested for their ability to with stand increasing concentrations of phorate ranging from $0-500 \mathrm{ppm}$ with a treatment interval of $100 \mathrm{ppm}$. Luria broth, inoculated with individual rhizobacteria and without phorate $(0 \mathrm{ppm})$ was considered as the positive contro i.e., (culture with ideal growth) against which the tolerance of respective rhizobacterial isolates to increasing phorate concentrations $(100-500 \mathrm{ppm})$ was tested. To $10 \mathrm{ml}$ of sterilized LB media, phorate was added from the stock solution, to make up the desired treatment concentrations. Constant amount of microbial inocula (RBI1, RBI2, RBI3 and RBI4), as mentioned in the previous section were added separately to each of the phorate supplemented test tubes aseptically and incubated overnight at $30 \pm 2{ }^{\circ} \mathrm{C}$ and $120 \mathrm{rpm}$ in orbital shaker (innova42 New Brunswick Eppendorf, Germany). Post incubation, absorbance was read at $595 \mathrm{~nm}$ (UV-1800 spectrophotometer, (Shimadzu Corp. Japan). The experiment was carried out in triplicate and the response of isolates to increasing phorate concentration is depicted in figure 1. Results for the same calculated based on formula (Shaheen and Sundari, 2013; Yadav and Sundari, 2015), as given below and tabulated (table 1):

$\%$ Survivability $=\frac{\text { Absorbance of treatment }}{\text { Absorbance of control }} \times 100$

* Considering growth in control as $100 \%$.

\section{Minimum Inhibitory Concentration (MIC) and Lethal dose $\left(\mathrm{LD}_{50}\right)$ values}

In order to assess the probable $\mathrm{LD}_{50}$ and MIC values of rhizobacterial isolates towards phorate, calculations were made by extrapolating the tolerance studies graph (Fig. 1). Experiments were further conducted (as explained in tolerance studies) where phorate concentrations was adjusted near extrapolated $\mathrm{LD}_{50}$ and MIC values. Inoculation was done with RBI1-RBI4 as explained in tolerance studies. Post incubation viability assay was performed taking $100 \mu \mathrm{l}$ of each sample in the wells of an ELISA plate, to which $10 \mu \mathrm{l}$ of freshly prepared stock of MTT [3-(4,5-Dimethylthiazol-2-yl)-2,5-Diphenyltetrazolium Bromide] (Himedia) was added. The samples were then incubated and checked for appearance of purple violet crystals (a positive indication of growth), which was further quantified after adding $100 \mu 1$ of DMSO (di-methyl sulfoxide) and absorbance measured at $570 \mathrm{~nm}$ using ELISA reader (Benchmark, Bio-Rad) (Shaheen and Sundari, 2013). The $\mathrm{LD}_{50}$ and MIC values thus obtained were tabulated (table 1).

\section{Substrate exclusion studies to establish Phorate metabolism}

Phorate metabolism studies were performed using specially designed media hereon referred to as PMB Media (in short for Plant Microbe bioassay Media), containing the salts: $\mathrm{CaCl}_{2} .2 \mathrm{H}_{2} \mathrm{O}, \mathrm{NaCl}, \mathrm{MgSO}_{4} .7 \mathrm{H}_{2} \mathrm{O}, \mathrm{KH}_{2} \mathrm{PO}_{4}, \mathrm{FeCl}_{3},\left(\mathrm{NH}_{4}\right)_{2}$ $\mathrm{HPO}_{4}$. Sodium citrate $(1 \%)$ was provided as the carbon source where applicable. Carbon and phosphate exclusion studies followed hypothesis as suggested by authors Rozo et al. (2010) and Shaheen and Sundari (2013). The experiment was conducted in two sets to represent carbon (C) exclusion (Set1) and phosphorous (P) exclusion (Set2) studies respectively. Set1 was designed to exclude readily available carbon source in place of which phorate was provided as the sole carbon source at five different concentrations: 100 ppm, 200 ppm, 300 ppm, $400 \mathrm{ppm}$ and $500 \mathrm{ppm}$ respectively. The experimental setup included two controls namely: $\mathrm{C} 1$ (containing $1 \%$ sodium citrate which served as positive control) and $\mathrm{C} 2$ (containing neither carbon nor pesticide representing the negative control). Set2 was designed for phosphate exclusion, where phorate at concentrations as mentioned above was provided as sole phosphate source and two controls namely: P1 (containing $1 \%$ potassium dihydrogen phosphate as phosphate source) and P2 (containing neither phorate nor potassium dihydrogen phosphate) were placed. To prepare the rhizobacterial inoculum, isolates RBI1, $\mathrm{RBI} 2, \mathrm{RBI} 3$ and RBI4 were grown on LB overnight under aseptic conditions and harvested the next day by centrifugation at $4,000 \mathrm{rpm}$ for $20 \mathrm{~min}$ at $4{ }^{\circ} \mathrm{C}$ (Hermal cold centrifuge). The bacterial pellet thus obtained was washed in two changes of saline solution followed by suspension of the pellet in $10 \mathrm{ml}$ of double sterilized distilled water. Every treatment of Set 1 and Set 2 contained $9 \mathrm{ml}$ of minimal media and $1 \mathrm{ml}$ of resuspended bacterial cell biomass added aseptically. All the test tubes from Set 1 and Set 2 were then incubated at $30 \pm 2{ }^{\circ} \mathrm{C}$ maintaining the rotation at $120 \mathrm{rpm}$. Isolates growth was then evaluated by performing viability assay as mentioned earlier, where samples were drawn ou aseptically following an incubation period of $24 \mathrm{hrs}$. Similar procedure was followed for both sets of experiments and the results depicted in figure 2 and 6 .

\section{Sample extraction for FTIR Analysis}

Based on the results from tolerance study, common concentration threshold was identified and all subsequent experiments (FTIR and HPLC) were carried out at threshold concentration. The experimental setup consisted of four treatments and a control as defined below. Un-inoculated LB broth with 300 ppm phorate supplementation was taken as the control while the four treatments were individually cultured rhizobacterial species (RBI1, RBI2, RBI3 and RBI4) and aseptically inoculated in the broth. Incubation was done for $48 \mathrm{~h}$ at $30^{\circ} \mathrm{C}$ and 120 rpm. Post incubation the culture soup was centrifuged at $6000 \mathrm{rpm}$ for $30 \mathrm{mins}$. Supernatant was extracted thrice with chloroform based on protocol suggested by Kadam and Gangawane (2005). Resultant extracts were pooled together and kept for drying at room temperature. Post drying samples were analysed through Fourier transformation infrared spectroscopy (FTIR) analysis. IR spectral study was done on FTIR-ATR instrument, Bruker Germany equipped with diamond crystal at Noble analytical labs, New Delhi India. IR spectra were recorded at room temperature (RT) in the frequency range of $1,700-500 \mathrm{~cm}^{-1}$ as represented in figures 3 and 4 . To avoid cross contamination amongst samples, the sample space was cleaned with isopropanol (IP) after every reading.

\section{Sample preparation for HPLC Analysis}

Phorate degradation by representative rhizobacterial isolate was studied using High performance liquid chromatography (HPLC). HPLC was performed both with control and a rhizobacteria treated sample. PMB media containing threshold phorate concentration along with rhizobacteria treatment was referred to as RBIT and media containing only phorate without rhizobacterial treatment served as control. Incubation was done for $48 \mathrm{hr}$ at $30^{\circ} \mathrm{C}$ and $120 \mathrm{rpm}$. Post incubation, extraction of phorate residues was done by pelleting out bacterial biomass through centrifugation, followed by residue extraction with equal volume of HPLC grade chloroform, three times as given by Kadam and Gangawane (2005). Extracts were then pooled and kept for drying at room temperature. Post drying sample was re-dissolved in $1 \mathrm{ml}$ chloroform. Both RBIT and control sample so prepared were then injected into the C-18 column of HPLC instrument (model LC 2010 Shimadzu Corp. Japan with UV-Visible detector and quaternary auto sampler). Separation was achieved using methanol and water as mobile phase in the ratio $70: 30$. Flow rate was set to $1 \mathrm{ml} / \mathrm{min}$ and absorbance was read at $230 \mathrm{~nm}$ (figure 5). Percent degradation of phorate was analysed by calculating the reduction in the peak area and intensity (height of peak) of RBIT as compared to phorate peak in control using the formula (2) given below.

$\%$ Degradation $=\frac{\text { Total Area } * \text { of major peak in RBIT }}{\text { Total Area } * \text { of major peak of Phorate in control }} \times 100$

* Total area $=$ area $\mathrm{x}$ height 


\section{RESULTS AND DISCUSSION}

\section{Phorate tolerance studies}

The principle aim of the study was to obtain most suitable rhizobacteria that has potential to degrade /metabolise phorate. Conventionally phorate is applied at high concentrations in agriculture soils i.e. 7.5 to $10 \mathrm{~kg}$ per acre (Gupta et al., 2015). However, the recommended phorate application dosage for Indian agriculture soils is approximately $1.5 \mathrm{~kg} / \mathrm{ha}$ (Kodandaram et al., 2016). Residual concentrations of phorate in soils differ based on type of soils and variety of crop plants. Studies done on different soils such as loam, peaty loam, sandy loam, muck etc revealed that muck soil has maximum phorate residue $(22.9-24.5 \mathrm{ppm})$ followed by silt loam (19.5-20.6 ppm) while the sandy loam soil possessed least residual phorate (Suett, 1971; Singh and Singh, 1984; Szeto et al., 1990) Singh and Singh (1984) also reported variations in phorate persistence and movement based on rate of phorate application. Between two different dosages viz., $4 \mathrm{~kg} / \mathrm{ha}$ and $8 \mathrm{~kg} / \mathrm{ha}$, higher persistence and mobility of phorate was observed at higher concentration i.e., $8 \mathrm{~kg} / \mathrm{ha}$. These authors also reported difference in phorate residual concentration with respect to season showing higher persistence in winter (29\% phorate residue) as compared to summers (17\% phorate residue) even after 90 days.

Since the study aims is to check microbe assisted remediation as a means to counter the problem of residual pesticides, accumulated in agriculture soil it is important to evaluate the stability and survivability of competent bacterial isolates at pesticide concentrations that exist in agriculture soil. Moreover, at the time of pesticide application, pesticide concentration will be very high in agriculture soil, which over a period of time would get variously diluted to a residual value. Competent rhizobacterial will have to survive through these wide variations in pesticide concentrations. Considering these facts, the first experiment was designed to explore the ability of native rhizobacterial isolates to survive a higher order of phorate concentration ranging from $100 \mathrm{ppm}$ to 500 ppm under in-vitro conditions. Growth response of rhizobacterial isolates RBI1, RBI2, RBI3 and RBI4 is plotted using Origin 8.1 software (Fig. 1). Bacterial survival was calculated as percentage survivability (table 1) using formula 1 given in materials and methods.
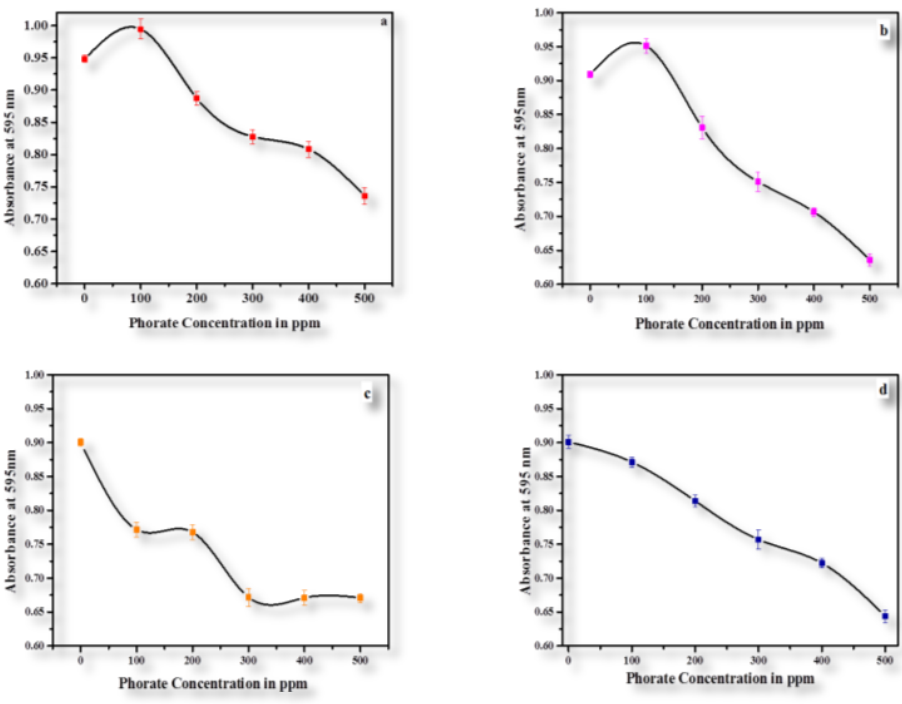

Figure 1Tolerance of rhizobacterial isolates to increasing Phorate concentration a) RBI1 b) RBI2 c) RBI3 and d) RBI4

From figure 1, it is evident that amongst the four native isolates studied, RBI1 and RBI2 have a better hold on tolerating initial OPP (phorate) stress. Both the isolates showed an increased (compared to control i.e. $0 \mathrm{ppm}$ ) growth at $100 \mathrm{ppm}$ phorate (Fig.1, table 1). This increment (though is marginal and not statistically significant), it is nonetheless a very encouraging phenomenon, observed in most of the resistant strains. In such strains spike in stress causes activation in the internal cellular metabolism and the cells respond by increasing their number or multiplication rate. Similar observations was made by earlier studies by Das $\boldsymbol{e}$ al., (1995) and (2003), stating that pesticides and certain chemical stressors may stimulate bacterial proliferation and bacterial count.

Beyond $100 \mathrm{ppm}$, all isolates registered a decrease in bacterial growth which continued further till $500 \mathrm{ppm}$ phorate. At $500 \mathrm{ppm}$, survival of RBI1 is $10 \%$ higher i.e. $79 \%$ than RBI2 (69\% survivability) proving it to be comparatively better contender to tolerate higher phorate concentration (statistically significant difference at p-value less than 0.05). RBI3 exhibited sensitivity to phorate as compared to other isolates. This decrease in growth can be particularly due to the release of phorate sulfoxide, a phorate degradation metabolite which may cause toxicity to sensitive bacterial strains (Venkatramesh et al., 1987, Barman and Das, 2015). Earlier studies with different herbicide/pesticide stress also reported decrease in microbial growth at higher concentrations of respective chemicals (Omar, 2001; Muturi et al., 2017). Although \% survivability of RBI3 at increasing OPP concentration is much less in comparison with other isolates but it is interesting to note that beyond $300 \mathrm{ppm}$, further increase in OPP concentration appears to have little impact on the isolate. Percentage survival in RBI3 has almost plateaued from $300(75.4 \%)$ to 500ppm (73.5\%). Also when the slope of tolerance was calculated, significant observation can be made with respect to the relative impact of each culture to increasing phorate concentrations While isolate RBI2 exhibited steeper slope (0.063), followed by RBI4 (0.0495) and RBI1 (0.048), least with RBI3 (0.0451). However difference in slope values from RBI1 and RBI4 in not significant. Hence isolate RBI3 may not be out ruled from its possible candidature for phorate remediation. RBI4 showed $97.8 \%$ survivability at phorate concentration of $100 \mathrm{ppm}$ and emerged as an equa contender with RBI1 registering $73 \%$ survivability at $500 \mathrm{ppm}$. At $300 \mathrm{ppm}$ phorate, all isolates showed comparable growth impact registering survival as 87.3\% (RBI1), $83.3 \%$ (RBI2), 75.4\% (RBI3) and 83.6\% (RBI4) respectively. All in all, the study can safely document that at $500 \mathrm{ppm}$ phorate concentration, (which is nearly 150 times to residual concentrations reported in agriculture soils) all native isolates still retained more than $70 \%$ survivability. As per author's observation, the maximum phorate concentration explored in any of the earlier degradation studies, was $\geq 100$ ppm (Bano and Musarrat, 2003; Kadam and Gangawane, 2005; Rani et al., 2009; Rani and Juwarkar, 2012). Jariyal et al. (2015a, b) was the only study where potential of Pseudomonas sp. and Brevibacterium frigoritolerans were studied for degrading phorate at concentrations upto $300 \mathrm{ppm}$ in soil. Present study puts forth an important observation where the native rhizobacterial isolates showed tolerance to higher phorate stress. Considering that beyond $300 \mathrm{ppm}$, majority of the isolates registered a steep decrease in survival, authors considered $300 \mathrm{ppm}$ as the threshold concentration at which further study on comparative phorate degradative ability of isolates.

\section{LD $_{50}$ and MIC studies}

Before embarking on specific degradation abilities, authors proceeded to determine the extreme limits of tolerance in RB isolates towards phorate (MIC) and their $\mathrm{LD}_{50}$. All the rhizobacterial isolates showed $\mathrm{LD}_{50}$ values in range 700 $1000 \mathrm{ppm}$ and MIC between 1450-2010 ppm respectively (table 1). Table 1 represent both extrapolated $\mathrm{LD}_{50}$ values calculated from tolerance study graphs $\left(\mathrm{R}^{2}\right.$ value $\left.0.96-0.99\right)$ and verified experimentally for accuracy. MIC values depicted in the table were experimentally derived after 3-4 alterations.

\begin{tabular}{lcccc}
\multicolumn{5}{c}{ Table 1 MIC and $\mathrm{LD}_{50}$ values of all rhizobacterial isolates } \\
\hline Isolates & $\begin{array}{c}\text { Impact of } \\
\text { survival at } \\
\text { 100ppm } \\
\text { survival at } \\
\text { conc. 150 } \\
\text { times higher } \\
\text { than in soil } \\
\text { residue } \\
\text { (500ppm) }\end{array}$ & $\begin{array}{c}\text { LD }_{\mathbf{5 0} \text { values }} \\
\text { (ppm) }\end{array}$ & $\begin{array}{c}\text { MIC values } \\
\text { (ppm) }\end{array}$ \\
\hline RBI1 & $104.8 \%$ & $77.6 \%$ & 950 & 1750 \\
RBI2 & $104.2 \%$ & $68.8 \%$ & 700 & 1450 \\
RBI3 & $85.3 \%$ & $73.5 \%$ & 1000 & 2010 \\
RBI4 & $97.8 \%$ & $73 \%$ & 900 & 1600 \\
\hline
\end{tabular}

Upon observing $\mathrm{LD}_{50}$ and MIC the apprehension that RBI3 can be a deserving candidate for phorate remediation was reaffirmed (table 1). RBI3 showed highest $\mathrm{LD}_{50}$ and MIC values: $1000 \mathrm{ppm}$ and $2010 \mathrm{ppm}$ respectively. Even in the tolerance study, barring the initial drop in growth, this culture showed resilience towards increasing phorate concentration (Fig. 1, table 1). This could be due to the fact that over a period of time, bacterial isolate start to degrade the toxic intermediates/metabolites and start utilizing them as substrate showing growth (Venkatramesh et al., 1987). Furthermore, authors have reported in the past highest emulsification index of $14 \%$ along with strong biofilm production in isolate RBI3 (Yadav and Sundari, 2015). These two physiological characteristics might be providing endurance in RBI3 to higher phorate concentrations (Singh et al., 2006; Calvo et al., 2009 ). RBI1 showed the second highest $\mathrm{LD}_{50}$ and MIC values (950 ppm and $\left.750 \mathrm{ppm}\right)$. Author's isolate RBI1 was already reported for strong ability to produce biosurfactants and biofilm (Yadav and Sundari, 2015), which not only help in solubilising the degradation-reluctant compounds but also protects bacterial cells against higher OPP concentration (Singh et al., 2006; Buchan, Gonzalez, 2010). Isolate RBI2 showed maximum sensitivity to increasing phorate stress, recording a drop $>30 \%$ in survival and lowest $\mathrm{LD}_{50}$, MIC values. This may be due to accumulation of degradation intermediates/metabolites proving toxic to RBI2 (Venkatramesh et al., 1987, Omar, 2001).

RBI4 by far can be placed as third best contender to endure increasing phorate stress To the best of author's knowledge this manuscript is the first report recording highest $\mathrm{LD}_{50}$ and MIC in native plant growth promoting rhizobacteria. Higher tolerance in these isolates can prove to be an important asset, which in future can be explored for remediation of phorate contaminated sites / 
agriculture soils freshly sprayed with phorate, where soil particles hold much higher concentration of residual phorate.

\section{Substrate exclusion studies}

Substrate exclusion study was designed to understand the metabolic mechanism employed by rhizobacterial isolates to degrade phorate. For chemical sources for carbon $(\mathrm{C}) /$ phosphate $(\mathrm{P})$, were replaced with phorate. Phorate utilization by the microorganisms was measured based on their survival in the absence of $\mathrm{C} / \mathrm{P}$ and $\%$ use efficiency with respect to phorate. Growth of native isolates was discernible at all phorate concentrations (ranging 100-500 ppm) tested (figure 2).

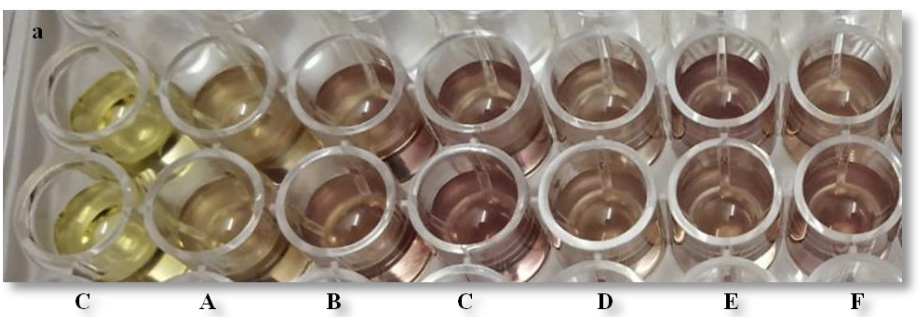

Figure 2 Assay showing positive growth of native isolate upon phorate supplementation

C: Control-1; A: Control-2; B: 100 ppm; C: 200 ppm; D: 300 ppm; E: 400 ppm; F: 500 ppm of Phorate concentration respectively.

Isolates RBI1, RBI4 and to some extent RBI3 could catabolise phorate as sole P source. In fact amongst all the isolates, RBI1 and RBI3 were found to metabolise phorate both as carbon and phosphate source while others leaned on phorate more as a carbon source. Of all the isolates, RBI2 least inclination to metabolise phorate neither as $\mathrm{C}$ nor as $\mathrm{P}$ source. RBI1 showed maximum growth efficiency of $156 \%$ at $300 \mathrm{ppm}$ of phorate and $87 \%$ growth efficiency at $500 \mathrm{ppm}$ of phorate as principle carbon source. When phorate was provided as $\mathrm{P}$ source, RBI1 recorded $95 \%$ growth efficiency at $100 \mathrm{ppm}$ and $81 \%$ growth efficiency at 500 $\mathrm{ppm}$. At higher concentrations (500 ppm) RBI3 had partial/very little preference towards utilization of phorate as $\mathrm{P}$ source and no preferential utilization of phorate as $\mathrm{C}$ source. $\mathrm{RBI} 3$ recorded a maximum of $62 \%$ growth efficiency with phorate as P source at $200 \mathrm{ppm}$ after which a steep drop in growth was observed with $5.9 \%$ utilization at $300 \mathrm{ppm}$ of phorate. RBI4 displayed prominent preference for phorate as P source with maximum efficiency of $177 \%$ at $100 \mathrm{ppm}$ followed by a decrease in percentage use efficiency registering $156 \%$ efficiency at $200 \mathrm{ppm}$ and $35 \%$ efficiency at $500 \mathrm{ppm}$ of phorate respectively. Higher concentrations of phorate $(500 \mathrm{ppm})$ proved to decrease isolates effeciency in metabolising phorate. The probable reason for this may be due to reported toxicity of primary degradation metabolite of phorate believed to be 100 fold more toxic than the parent compound itself (Bano and Musarrat, 2003; Rani $\boldsymbol{e}$ al., 2009). RBI3 and RBI4 were noticed to metabolise phorate preferably as sole $\mathrm{P}$ source. Microbes were previously reported to show lesser inclination for given pesticide as two substrate source and a greater preference as one substrate source (Singh and Walker, 2006). Study by Claassen, (1988) and Cook et al. (1978) provide evidence regarding utilization of organic phosphorous compounds as sole $\mathrm{P}$ source by microbes. In certain studies, Rhizobium sp., Pseudomonas sp., Proteous sp. and Ralstonia eutropha were reported to utilize phorate as sole C source (Bano and Musarrat, 2003; Rani et al., 2009). Results from the study are in accordance with earlier observations where bacterial isolates were found to metabolise pesticides by using it as single nutrient source preferably ( Bano and Musarrat, 2003; Singh and Walker, 2006; Singh and Walker, 2006; Rani $e t$ al., 2009).

Substrate exclusion studies made it clear that the native isolates can utilise phorate either as ( $\mathrm{C}$ or $\mathrm{P}$ ) suggesting isolate specific mode of metabolism alternating between catabolism and co-metabolism. Several studies propose microbes for efficient degradation of different classes of pesticides with ability to utilize the chemical as principle substrate. Such instances where the chemical/pesticide is metabolised in the presence other complex or preferential substrates (C/N/P) are referred to as performing co-metabolism (Singh and Walker, 2006; Bano and Musarrat, 2003; Rani et al., 2009). Since RBI2 was found to show preference neither as $\mathrm{C}$ nor as a $\mathrm{P}$ source, it can be inferred that RBI2 may metabolise phorate through co-metabolism. Figure 3 presents an argument on isolate specific preference towards phorate degradation. At lower concentration $(100 \mathrm{ppm})$, all isolates exhibited ability to metabolise phorate either as $\mathrm{C}$ or $\mathrm{P}$ source. However, as the concentration of phorate increased, only two isolates RBI1 and RBI4 showed sustained use efficiency. Another important observation from figure 3 is about the significance of incorporating $\mathrm{C}$ and $\mathrm{P}$ exclusion studies in determining the relative efficiency of isolates. In the absence of $\mathrm{C}$ and $\mathrm{P}$ exclusion data, the figure would have presented an altogether different orientation leading to biased conclusion. Thus comparing and contrasting all 5 parameters i.e., Tolerance, $\mathrm{LD}_{50}$, MIC, phorate as $\mathrm{C}$ source and phorate as $\mathrm{P}$ source, the trend in degradative abilities over increasing phorate can be summarised as follows RBI4 - at low phorate and P deficient conditions, RBI1 under balanced $\mathrm{P}$ and $\mathrm{C}$ and also with extreme phorate toxicity. The strong point in favour of present study is isolate's greater metabolic potential towards OPP and ability to catabolically breakdown phorate at much higher concentrations.
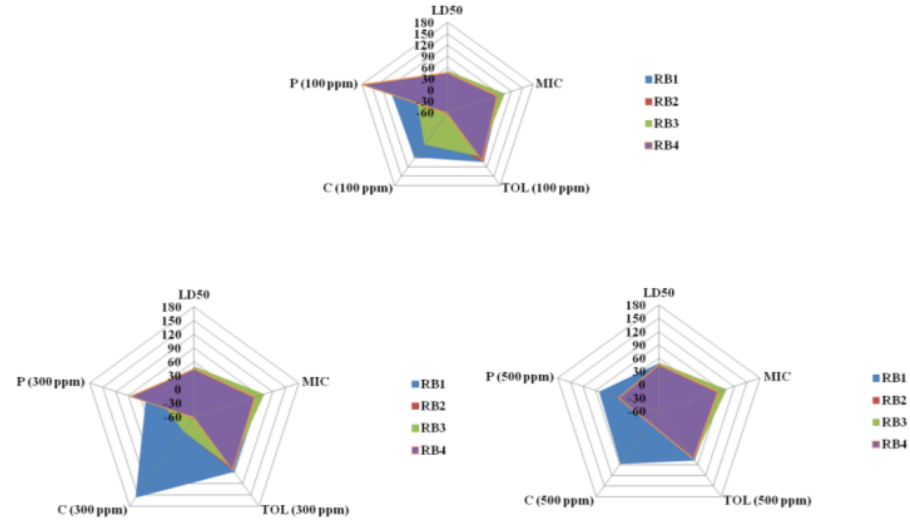

Figure 3 Parameter comparison for phorate degradation at a) $100 \mathrm{ppm}$; b) 300 ppm; c) 500 ppm

\section{FTIR analysis}

FTIR is an IR spectroscopy technique which provides more detailed information about the chemical structure of compounds via functional group identification (Rani and Juwarkar, 2010). This technique can also point structural changes and transformation occurring in any parent compound under study as a result of different treatments. Phorate degradation ability of isolates was explored in the present study at a threshold concentration of $300 \mathrm{ppm}$, a concentration that is approximately hundred times higher than reported residual limit (20-30 ppm) in soils. Through FTIR analysis degradation metabolites (intermediates, end products) of phorate were observed. FTIR spectroscopy for phorate standard and degradation samples was done at finger print range, (wave number ranging from 1800-600 $\mathrm{cm}^{-1}$ ) on FTIR-ATR instrument. FTIR spectrum obtained for the standard is shown in figure 3. Peaks observed in the standard spectrum at 1025 $\mathrm{cm}^{-1}$ indicate the presence of ester linkages as is expected, since phorate is an OPP containing esters of phosphoric acid. Another peak at $998.37 \mathrm{~cm}^{-1}$ and $908.99 \mathrm{~cm}^{-1}$ can be categorized as alkenes (C-H linkages). Similarly peak in the range of $1050-990 \mathrm{~cm}^{-1}$ indicates the presence of P-O-C linkages in standard which is one of the major linkages in phorate. Peak at $777.40 \mathrm{~cm}^{-1}$ and 747.86 $\mathrm{cm}^{-1}$ depicts alkynes and standard peak at $676.73 \mathrm{~cm}^{-1}$ and $624.11 \mathrm{~cm}-1$ shows presence of $\mathrm{C}-\mathrm{S}$ stretch that can be either $\mathrm{CH}_{3}-\mathrm{S}$ - or $\mathrm{CH}_{2}=\mathrm{S}$ linkages which can be observed in phorate structure (QI et al., 2008; Coates 2000; Beauchamp 2004) (Fig. 4).

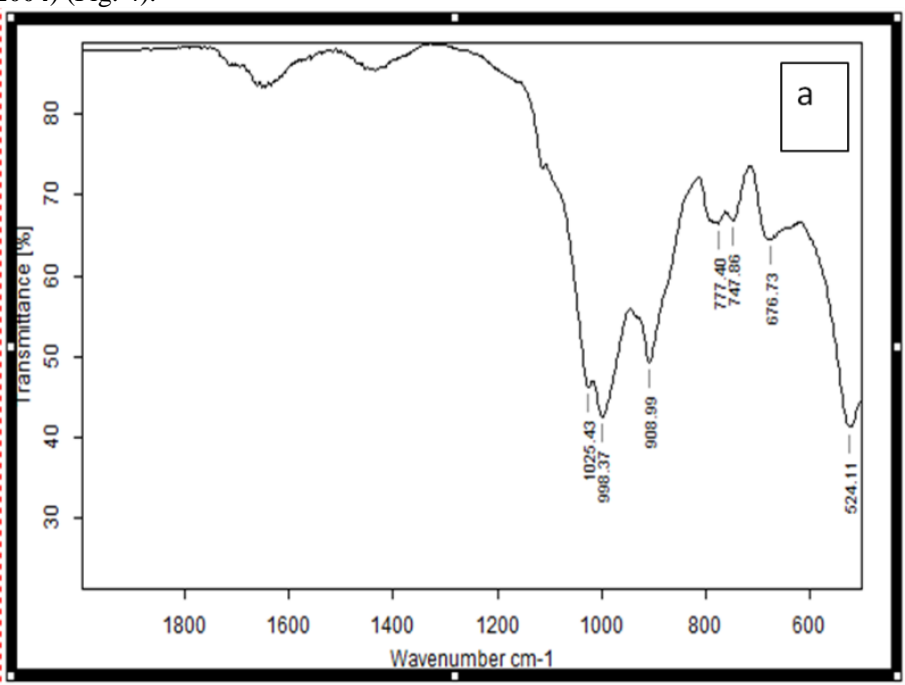

Figure 4 FTIR spectrum of Phorate standard 


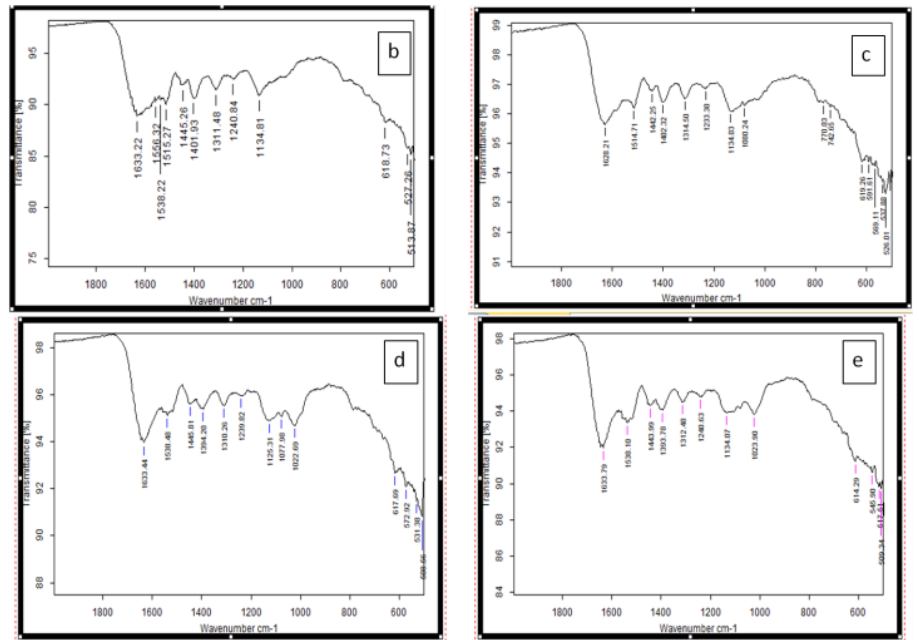

Figure 5 FTIR spectrum of residual phorate upon rhizobacterial treatment for $48 \mathrm{hrs}$

b: RBI1 treated sample; c: RBI2 treated sample; d: RBI3 treated sample; e: RBI4 treated sample

In the present study FTIR was explored to understand the transformation occurring in phorate structure as a result of rhizobacterial treatment. Structural changes in the spectrum brought about due to degradation of phorate by different isolates were visibly comparable (Fig. 5). Difference in \% transmittance can also be observed on comparing IR spectra of phorate standard to that of rhizobacterial treated samples (Fig. 4 and Fig. 5). FTIR spectra of phorate subjected to rhizobacterial treatment showed disappearance of major bands at 1025.43, 998.37 and $908.99 \mathrm{~cm}-1$, as compared to phorate standard (Fig. 5). Formation of new IR bands was observed in the degraded samples at wave numbers 1633,1538 , 1528, 1556, 1538 and $1514 \mathrm{~cm}^{-1}$, which could be due to formation of C-C linkages and bending or deformation of $\mathrm{C}=\mathrm{C}$ bond (Fig. $5 \mathrm{a}, \mathrm{b}, \mathrm{c}$ and d). Bands observed in the range $1400-1450 \mathrm{~cm}^{-1}(1401,1402,1442,1443,1445), 1393$, 1310 and $1314 \mathrm{~cm}^{-1}$ (QI et al., 2008; Coates, 2000; Beauchamp, 2004) indicate presence of methyl C-H stretch, tertiary $\mathrm{O}-\mathrm{H}$ bend and organic sulphate respectively. Organic phosphate bonds were observed at $1350 \mathrm{~cm}^{-1}$ wave number Similarly IR bands observed at $1240,1134,1125$ and $1080 \mathrm{~cm}^{-1}$ wave numbers indicate: $\mathrm{C}-\mathrm{H}$ bond stretch, presence of organic phosphate, suphonate and aryl sulphone respectively. Also the degradation samples peaks at $645,614,618,770$ and $740 \mathrm{~cm}^{-1}$ indicate mono and di-substitutions.

Shift in the peak or reduction in the peak intensity along with formation of new peaks as observed in the present study indicates either dissociation of parent compound (Phorate) or change in the parent compound structure leading to formation of new degradation intermediates or end products (Jain and Garg, 2013). The pattern of results obtained was comprehended and analysed by referring to several previous studies targeting other OPP degradation studies that applied FTIR (Pehkonen and Zhang 2010; Jain and Garg, 2013; Neti and Zakkula, 2013). As ester linkages form the basis of OPP structure, dissolution of major standard peak corresponding to ester linkage $\left(1025 \mathrm{~cm}^{-1}\right)$ observed in the study was a significant indicator of phorate degradation by native rhizobacterial isolates. These results can also be justified by referring to previous studies that report the importance of ester bond hydrolysing enzymes in OPP degradation (Kadam and Gangawane, 2005; Ruggaber and Talley, 2006; Singh and Walker, 2006; Tallur et al., 2008; Sayali et al., 2013). Moreover, change in $\%$ transmittance as observed between standard and sample (Fig. 4, 5) suggests reduction in the intensity of phorate peak in RB treated sample. Previously FTIR analysis for phorate has been done to explore soil and phorate interaction studies (Rani and Juwarkar, 2010). To the best of author's knowledge this is the first study where FTIR spectroscopy is attempted to understand phorate degradation by pure bacterial cultures in-vitro (under controlled conditions), leaving no room for any ambiguity on who is contributing for the breakdown of phorate.

\section{HPLC Analysis}

Post rhizobacterial treatment, the cell free soup was subjected to HPLC analyses. From results of HPLC analysis (Fig. 6), it was inferred that $42 \%$ of phorate was degraded by native rhizobacterial isolate where the initial concentration was 300 ppm. Barring the study by (Jariyal et al., 2015a, b), not many studies attempted phorate degradation at concentrations higher than $100 \mathrm{ppm}$. In vitro studies targeting degradation of phorate at higher concentrations are few and far between. Being water soluble, phorate would progressively leach out from soil microcosms leaving minimal residue in soil over a period of time. Half life of phorate in soil is reported to be between 2-173 days, which is a fairly large window. Hence there is a requirement for total in-vitro assessment of phorate residue in the medium post inoculation with microbes where every parameter like media, salts, substrate concentration, isolate type, and dosage can all be regulated. For instance, study by Bano and Musarrat, 2003 reports 98\% degradation of phorate at a concentration of $200 \mathrm{ppm}$ in 40 days by Rhizobium, Pseudomonas and Proteous in soil. Their study does not elaborate on experimental set-up except for stating that it is a microcosm study. Another study by Kadam and Gangawane, 2005 reports $44-51 \%$ degradation of phorate at $150 \mathrm{ppm}$ concentration in liquid media in 7 days by Azotobacter isolates. Research by Rani et al., 2009 reports $85 \%$ degradation of phorate at concentration of $20 \mathrm{ppm}$ in 10 days in liquid media by Ralstonia eutropha. In comparison to all the previous researches done to assess phorate degradation, the present study shows $42 \%$ degradation within $48 \mathrm{hrs}$ and at concentration higher than any of the previous reports studied under in-vitro conditions.

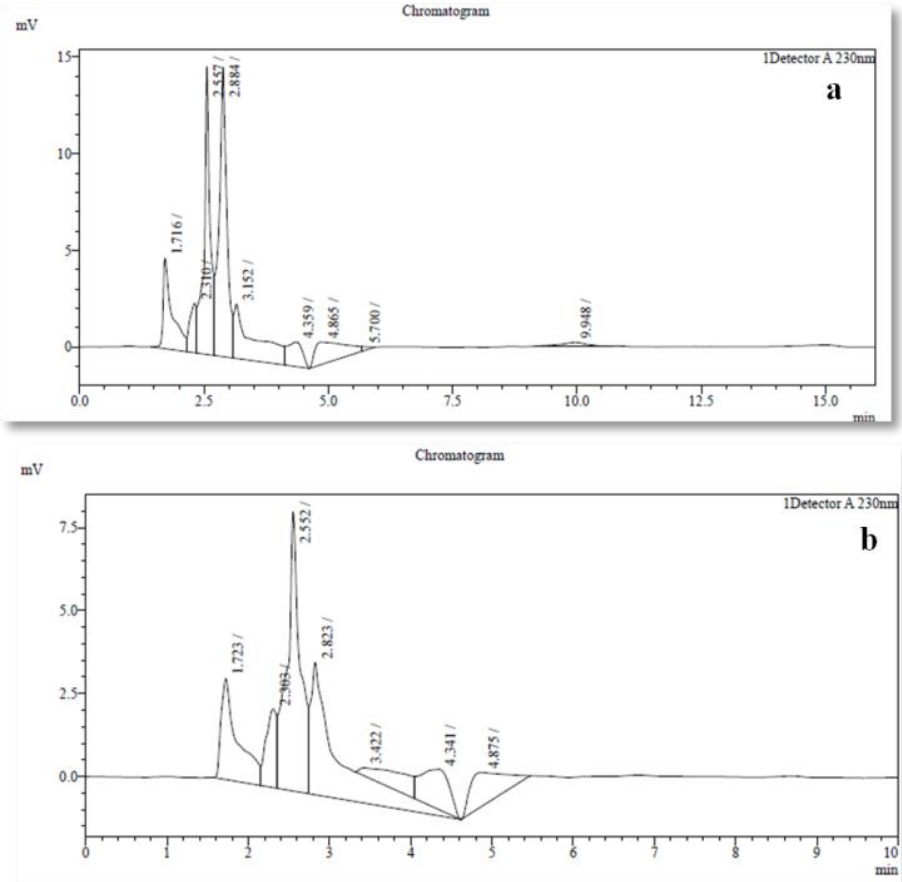

Figure 6 HPLC chromatogram showing degradation of Phorate (300ppm) upon treatment with Rhizobacteria RBI1 in $48 \mathrm{hrs}$

a)Phorate control; b) Rhizobacterial treated sample

The study presents a comparative assessment on the relative strengths of different rhizobacterial isolates employed to target phorate. Impact of increasing phorate concentration $(100 \mathrm{ppm}, 300 \mathrm{ppm}$ and $500 \mathrm{ppm})$ on four native rhizobacterial isolates (RBI1, RBI2, RBI3 and RBI4) can be summarised based on five parameters (namely: Tolerance, LD50, MIC, $\mathrm{C}$ exclusion and $\mathrm{P}$ exclusion) as represented concisely in figure 3 . In Indian agriculture soils where phosphorous is a limited availability resource, isolate RBI4 would be the most appropriate choice. This is because RBI4 not only degrade phorate but also metabolising P which can be a value addition for plant nutrition and growth. At very high concentrations like $500 \mathrm{ppm}$ phorate, in pesticide intoxicating conditions with marginal nutrient levels, isolate RBIl can be employed for phorate remediation and detoxification. However considering that Indian soils are $\mathrm{P}$ deficient and also because low phorate in study would mean $100 \mathrm{ppm}$ (which is already 4-5 times higher than reported residual phorate in agricultures soils), isolate RBI4 can be the isolate of choice for Indian agriculture soils.

\section{CONCLUSION}

Higher phorate toxicity, its persistence and sustained activity post application, drives the research concern towards neutralizing toxic phorate residues present in soil and environment. Through this study authors report potential of native rhizobacterial isolates RBI1, RBI2, RBI3 and RBI4 in metabolising phorate at 10 to 15 fold higher (300 ppm) residual phorate concentration as is present in agriculture soils. From this study it can be concluded that native isolates can convincingly survive on minimal salt media. In addition these isolates were also observed to utilize phorate preferentially as sole phosphate source at elevated phorate concentration $(500 \mathrm{ppm})$ rather than using it as sole carbon source. Earlier studies where FTIR was performed with phorate were majorly on determining phorate residue in soil and food samples. Thus author's study of phorate metabolism through FTIR is the first time report where phorate in the media was metabolised using pure rhizobacterial culture (in-vitro). Appearance of bands related to phorate degradation intermediates and end products is convincing evidence that supports the ability of these isolates to metabolise phorate. Conclusive evidence from HPLC confirms degradation of phorate at higher concentration of phorate by competent rhizobacteria within $48 \mathrm{hrs}$. All in all, the tested rhizobacterial isolates present a viable option for furthering research to develop farm-friendly bioinoculants. Formulation employing these 
isolates can be promoted for dual benefits of plant growth support and pesticide remediation.

Acknowledgments: Author would like to acknowledge the financial support provided by Department of Biotechnology (DBT), Ministry of Science and Technology, New Delhi, India. Role of Nobel Labs Pvt. Ltd., Delhi, India for providing facilities to conduct FTIR and HPLC analysis is appreciated. Support from Jaypee Institute of Information Technology for the conduct of research study is thankfully acknowledged.

\section{REFERENCES}

Abdollahi, M. \& Karami-Mohajeri, S. 2012. A comprehensive review on experimental and clinical findings in intermediate syndrome caused by organophosphate poisoning. Toxicology and Applied Pharmacology, 258(3), 09314

DOI: 10.1016/J.TAAP.2011.11.014

Antonijevic, B. \& Stojiljkovic, M. P. 2007. Unequal efficacy of pyridinium oximes in acute organophosphate poisoning. Clinical Medicine and Research, 5 , 71-82.

\section{DOI: 10.3121/CMR.2007.701}

Bano, N. \& Musarrat, J. 2003. Isolation and characterization of phorate degrading soil bacteria of environmental and agronomic significance. Letters in Applied Microbiology, 36, 349-353.

DOI: 10.1046/J.1472-765X.2003.01329.X

Barman, S. \& Das A. C. 2015. Residual effect of pre-emergence herbicides on microbial activities in relation to mineralization of $\mathrm{C}, \mathrm{N}$ and $\mathrm{P}$ in the Gangetic alluvial soil of West Bengal, India. Environmental Monitoring and Assessment, 187, 709-715

\section{DOI: $10.1007 /$ S10661-015-4698-9}

Beauchamp, P. (2004). Infrared Tables (Short summary of common absorption frequencies).

\section{HTTPS://WWW.CPP.EDU/ PSBEAUCHAMP/PDF/SPEC_IR_NMR_SPE}

\section{CTRA_TABLES.PDF}

Berny, P., Caloni, F., Croubels, S., Sachana, M., Vandenbroucke, V., Davanzo, F. \& Guitartet, R. 2010. Animal poisoning in Europe. Part 2: Companion animals. Veterinary Journal, 183, 255-259.

\section{DOI: 10.1016/J.TVJL.2009.03.03}

Buchan, A. \& Gonzalez, J. M. Handbook of hydrocarbon and lipid microbiology. 2010.

DOI: 10.1007/978-3-540-77587-4

Calvo, C., Manzanera, M., Silva-Castro G. A., Uad, I. \& Gonzalez-Lopez, J 2009. Application of bioemulsifiers in soil oil bioremediation processes. Future prospects. Science of the Total Environment, 407, 3634-3640.

DOI: 10.1016/J.SCITOTENV.2008.07.008

Chaudhary, T. \& Shukla, P. 2019. Bioinoculants for bioremediation applications and disease resistance : innovative perspectives. Indian Journal of Microbiology, $59,129-136$.

\section{DOI: 10.1007/S12088-019-00783-4}

Claassen, N. \& Tarafdar, J. C. 1988. Organic phosphorus compounds as a phosphorus source for higher plants through the activity of phosphatases. Biology and Fertility Soils, 5, 308-312.

DOI: 10.1007/BF00262137

Coates, J. (2006). Interpretation of infrared spectra, A practical approach Encyclopedia of analytical chemistry. DOI:10.1002/9780470027318.A5606.

Cook, A. M., Daughton, C. G. \& Alexander, M. 1978. Phosphorus-containing pesticide breakdown products: quantitative utilization as phosphorus sources by bacteria. Applied and Environmental Microbiology, 36, 668-672.

Das, A. C., Chakravarty, A., Sukul, P. \& Mukherjee, D. 1995. Insecticides: their effect on microorganisms and persistence in rice soil. Microbiological Research, 150, 187-194.

\section{DOI: $10.1016 / S 0944-5013(11) 80055-8$}

Das, A.C., Chakravarty, A., Sukul, P. \& Mukherjee, D. 2003. Influence and persistence of phorate and carbofuran insecticides on microorganisms in rice field. Chemosphere, 53, 1033-1037.

DOI: 10.1016/S0045-6535(03)00713-6

EL-Helow, E. R., Badawy, M. E. I. \& Mabrouk, M. E. M. 2013. Biodegradation of chlorpyrifos by a newly isolated bacillus subtilis strain Y242. Bioremediation journal, 17, 113-123.

DOI: $10.1080 / 10889868.2013 .786019$

Gangadhar, B., Tharakeswar, Y., Kalyan, Y. \& Naidu, G. R. 2016. Tea (Camellia Sinensis) waste for the reduction of malathion and phorate in aqueous solutions. International Journal of Environmental and Agriculture Research, 2, 38-44.

Gevao, B., Semple, K. T. \& Jones, K. C. 2000. Bound pesticide residues in soils A review. Environmental Pollution, 108, 3-14.

Gupta, S., Dangayach, S. \& Sundari, S. K. 2015. Investigating the role of PGPM in assisting plant growth under stress caused by organophosphate pesticidephorate. Indo Global Journal of Pharmaceutical Sciences, 5, 129-137.
Jain, R. \& Garg, V. 2013. Enzymatic degradation of monocrotophos by extracellular fungal OP hydrolases. Applied Biochemistry and Biotechnology, 171, 1473-1486.

\section{DOI: 10.1007/S12010-013-0438-1}

JARIYAL, M., GUPTA, V. K., JINDAL, V. \& MANDAL, K. 2015 A. Isolation and evaluation of potent Pseudomonas species for bioremediation of phorate in amended soil. Ecotoxicology and Environmental Safety, 122, 24-30.

DOI: 10.1016/J.ECOENV.2015.07.007

Jariyal, M., Gupta, V. K., Mandal, K. \& Jindal, V. 2015B. Brevibacterium frigoritolerans as a novel organism for the bioremediation of phorate. Bulletin of Environmental Contamination and Toxicology, 95, 680-686.

DOI: 10.1007/S00128-015-1617-2

Jariyal, M., Jindal, V., Mandal, K. Gupta, V. K. \& Singh, B. 2018 Bioremediation of organophosphorus pesticide phorate in soil by microbial consortia. Ecotoxicology and Environmental Safety, 159, 310-316.

DOI: 10.1016/J.ECOENV.2018.04.063

Kadam, T.A., Gangawane, L. V. 2005. Degradation of phorate by Azotobacter isolates. Indian Journal of Biotechnology, 4, 153-155.

Kalaivanan, N., Venkataramanan, R., Sreekumar, C., Saravanan, A. \& Srivastava, R.K. 2011. Secondary phorate poisoning of large carnivores in India. European Journal of Wildlife Research, 57, 191-194.

DOI: 10.1007/S10344-010-0433-6

Karami, M. S. \& Abdollahi, M. 2011. Toxic influence of organophosphate, carbamate, and organochlorine pesticides on cellular metabolism of lipids proteins, and carbohydrates: A systematic review. Human and Experimental Toxicology, 30, 1119-1140.

DOI: $10.1177 / 0960327110388959$

Kodandaram, M. H., Rai, S. S. A. B. \& Naik, P. S. 2016. Compendium on pesticide use in vegetables compendium on pesticide use in vegetables Indian institute of vegetable research. IIVR Extension Bulletin no. 50, ICAR, 1-132.

Maria, M., 2010. Phorate Toxipedia updated July 08 available at 〈http://toxipedia.org/ display/toxipedia/Phorate〉

Morowati, M. 1998. Inhalation toxicity studies of thimet (phorate) in the male swiss albino mouse, Mus musculus: ii. Lung histopathology, pseudocholinesterase level and haematological studies. Environmental Pollution, 103, 309-315

DOI: 10.1016/S0269-7491(98)00111-0

Muturi, E. J., Donthu, R. K., Fields, C. J., Moise, I. K. \& Kim, C. H. 2017. Effect of pesticides on microbial communities in container aquatic habitats. Scientific Reports, 7:1-10. DOI: 10.1038/SREP44565

Neti, N. \& Zakkula, V. 2013. Analysis of chlorpyrifos degradation by Kocuria $s p$. using GC and FTIR Nagavardhanam. Current Biotica, 6:466-472.

DOI: 10.1038/SREP44565

Omar, S. A. 2001. Microbial populations and enzyme activities in soil. Water Air, and Soil Pollution, 127, 49-63.

Qi, J., Shi, D., Jiang, X. 2008. The structures and electronic properties of doublewall bismuth nanotubes from first-principle calculations. Chemical Physics Letters, 460(1-3), 266-271.

\section{DOI:10.1016/j.cplett.2008.06.033.}

Pehkonen, S.O. \& Zhang, Q. 2010. The degradation of organophosphorus pesticides in natural waters: a critical review the degradation of organophosphorus pesticides in natural waters: A critical review. CriticaL Reviews in Environmental Science and Technology, 1:17-72.

\section{DOI: 10.1080/10643380290813444}

Rani, R. , Lal, R., Kanade, G.S. \& Juwarkar, A. 2009. Isolation and characterization of a phorate degrading bacterium. Letters in Applied Microbiology, 49:112-116.

DOI:10.1111/J.1472-765X.2009.02631.X

Rani, R. \& Juwarkar, A. 2010. Adsorption of phorate, an organophosphorus pesticide, on vertisol. Archives of Environmental Contamination and Toxicology, 58, 927-934.

DOI: 10.1007/S00244-009-9424-6

Rani, R. \& Juwarkar, A. 2012. Biodegradation of phorate in soil and rhizosphere of Brassica juncea (Indian mustard) by a microbial consortium. International Biodeterioration and Biodegradation, 71, 36-42.

\section{DOI: 10.1016/J.IBIOD.2012.04.004}

Ratna, Kumari, A., Sobha, K. \& Mounika, K. 2012. Molecular characterization of bacteria capable of organophosphate degradation. International Journal of Life Sciences Biotechnology and Pharma Research, 1, 64-72.

Rozo, C. J., Nieves, J. S., Vélez, D. U. \& Chacón, L. M. M. M. 2013. Characterization of carbofuran degrading bacteria obtained from potato cultivated soils with different pesticide application records. Rev Fac Nal Agr Medellín, 66, $6899-6908$

Ruggaber, T. P. \& Talley, J. W. 2006. Enhancing bioremediation with enzymatic processes: a review. Practice Periodical of Hazardous, Toxic and Radioactive Waste Management, 10 (2), 73-85.

DOI: 10.1061/(ASCE)1090-025X(2006)10:2(73)

Sayali, K., Sadichha, P. \& Surekha, S. 2013. Microbial esterases : An overview. Journal of Current Microbiology and Applied Science, 2, 135-146. 
Shaheen, S. \& Sundari, S.K. 2013. Exploring the applicability of PGPR to remediate residual organophosphate and carbamate pesticides used in agriculture fields. International Journal of Agriculture and Food Science Technology, 4 947-954.

Singh, Z. \& Singh, G. 1984. Persistence and movement concentrations of phorate at high in soil. Ecotoxicology and Environmental Safety, 8, 540-550.

Singh, N., Singh, B., Dureja, P. \& Sethunathan, N. 2003. Persistence of phorate in soils: role of moisture, temperature, preexposure and microorganisms. Journal of Environmental Science Health Biology, 38, 723-735.

DOI: 10.1081/PFC-120025556

Singh, R., Paul, D. \& Jain, R. K. 2006. Biofilms: implications in bioremediation. Trends in Microbiology, 14, 389-397.

DOI: 10.1016/J.TIM.2006.07.001

Singh, B.K. \& Walker, A. 2006. Microbial degradation of organophosphorus compounds. FEMS Microbiology Reviews, 30, 428-471.

DOI: 10.1111/J.1574-6976.2006.00018.X

Sørensen, S.R., Albers, C.N. \& Aamand, J. 2008. Rapid Mineralization of the Phenylurea Herbicide Diuron by Variovorax $s p$. Strain SRS16 in Pure Culture and within a Two-Member Consortium. Applied and Environmental Microbiology, 74, 2332-2340.

DOI: 10.1128/AEM.02687-07

Suett, D.L. 1971. Persistence and degradation of chlorfenvinphos, diazinon, fonofos and phorate in soils and their uptake by carrots. Pesticide Science, 2, $105-112$.

DOI: 10.1002/PS.2780020304

Szeto, S.Y., Price, P.M., Mackenzie, J.R. \& Vernon, R.S. 1990. Persistence and uptake of phorate in mineral and organic soils. Journal of Agricultural and Food Chemistry, 38, 501-504.

Tallur, P. N., Megadi, V. B. \& Ninnekar, H. Z. 2008. Biodegradation of cypermethrin by micrococcus sp. strain CPN 1. Biodegradation, 19, 77-82.

DOI: 10.1007/S10532-007-9116-8

US EPA (April 1998). Guidelines for ecological risk assessment. EPA/630/R95/002F, Federal Register 63(93):26846-26924).

Venkatramesh, M., Agnihothrudu, V., Mithyantha, M.S. \& Tripathi, S.C. 1987. Persistence of phorate in different soils with and without amendments and its degradation by a Pseudomonas sp. Bulletin of Environmental Contamination and Toxicology, 38, 381-387.

DOI: 10.1007/BF01606602

Yadav, I. C., Devi, N. L., Syed, J. H., Cheng, Z., L. I, J., Zhang, G. \& Jones, K. C. 2015. Current status of persistent organic pesticides residues in air, water, and soil, and their possible effect on neighbouring countries: A comprehensive review of India. Science of the Total Environment, 511, 123-137.

DOI: 10.1016/J.SCITOTENV.2014.12.041

Yadav, P. \& Sundari, S. K. 2015. Plant growth promoting rhizobacteria: An effective tool to remediate residual organophosphate pesticide methyl parathion, widely used in Indian agriculture. Journal of Environmental Research and Development, 9, 1138-1149. 